\title{
Complications and Failures of Implants
}

\section{Sonia Nanda ${ }^{1 *}$, Tarun Nanda² and Deepak Grover ${ }^{3}$}

${ }^{1}$ Department of Prosthodontics and Crown and Bridge, National Dental College, Derabassi, Punjab, India

${ }^{2}$ Department of Periodontics, Bhojia Dental College, Baddi, Himachal Pradesh, India

${ }^{3}$ Department of Periodontics, National Dental College, Derabassi, Punjab, India

\begin{abstract}
Although the high predictability and long-term success rate of dental implants is well documented, complications and failures do occur. Some complications may be relatively minor and easy to correct, while others will be major and result in the loss of the implant or prosthesis. The aim of this paper is to discuss complications and failures of dental implants.
\end{abstract}

Keywords: Implants; Bone; Traumatic occlusion, High-torque

\section{Introduction}

Since the introduction of the concept of osseointegration, the success of dental implants has increased dramatically because of better understanding of bone response and the improvement in bone loading concepts. Improper patient selection, accumulation of bacterial plaque because of poor oral hygiene, traumatic occlusion, debris retention resulting from improper prosthetic restoration, and bone preparation without the use of internally cooled, high-torque, slowspeed handpieces, have been the factors contributing to the breakdown of otherwise successful implants.

\section{Literature Review}

Various complications involve soft tissue, bone, and mechanical implant components [1]. Apart from this a major cause of failure is poor esthetics.

\section{Soft tissue complications}

Exposure of the cover screw: After the first stage surgery, the fixture installation site is checked for properly sutured wound closure. If the wound appears open, infection can occur, and inflammatory reactions can extend to the underlying fixture sites. After healing occurs, the tissues may pull away or shrink exposing cover screws. Residual suture material can cause an abscess or granulation tissue formation.

If cover screws are exposed, evaluate the cause such as inflammation or poor plaque control. When the exposure occurs within weeks after the first stage surgery, it may not be necessary to perform a covering procedure over the cover screw; it is dependent on the plaque-control factor. With poor plaque control or any other contraindications such as esthetics or infection, the cover screw should be covered surgically. This procedure requires removal of granulation tissue prior to repositioning gingival tissues [2]. A laterally positioned flap procedure is required when there is an inadequate amount of tissue present to cover the cover screws [3].

If attached mucosa is not available, a free gingival graft may be indicated to cover the cover screws [4]. To perform this procedure, the recipient site is prepared with a no. 15 blade by incising the marginal mucosa around the cover screw. A flap consisting of epithelium and underlying connective tissue is separated from the periosteum without damaging this layer. A partial thickness graft of masticatory mucosa approximately 1,0 to $1.5 \mathrm{~mm}$, is obtained from an edentulous ridge or the palate. The graft is placed over the recipient site and pressed into position with moist gauze. Suture the graft to the site with silk suture or resorbable, plain gut suture material to immobilize the tissue.
Protect the donor site by covering with tin foil initially then cover with tetracycline ointment; this combination helps inhibit bacterial growth and maintains donor tissue position. Next, place a periodontal pack for one week and repeat if necessary. Periodontal pack retention is enhanced if the patient wears a prosthesis. The tissue surface of the prosthesis is modified over the site, then the prosthesis can be seated over the periodontal pack.

The periodontal pack is removed after two weeks. Oral hygiene should be maintained during and after pack use. If a patient does not have a denture, a resin splint can be fabricated to protect the wound area.

A connective tissue graft is another procedure available to cover an exposed cover screw. The procedure is similar to the free gingival graft but differs in that only the connective tissue layer is removed from the donor site instead of a partial thickness graft.

Proliferative gingivitis: The peri-implant epithelium can occasionally grow around the abutment and exposed gold cylinder of the prosthesis, resulting from proliferative gingivitis [2]. This situation makes the sulcular area difficult to clean. A surgical procedure is necessary for removal of excess tissue. A gingivectomy or a flap operation with an internal beveled incision, is done to eliminate the pocket.

Exposure of fixture threads: The traumatic forces contributing to the inflammatory process and subsequent marginal bone loss sometimes result in exposed fixture threads. The incidence of this occurrence is less than $1.5 \%$ [2] and can happen when initially the fixture was not completely installed into the bone without proper countersinking procedures. The thread exposure may require a bone grafting procedure prior to periodontal treatment.

To prevent potential problems, plaque control should be reinforced in combination with a vestibuloplasty and a free gingival graft procedure, done to create attached gingiva for coverage of exposed fixture threads. The treatment of choice for this complication

*Corresponding author: Sonia Nanda, Department of Prosthodontics and Crown and Bridge, National Dental College, Derabassi, Punjab, India, Tel: 8607865955 ; E-mail: drsonia84@gmail.com

Received: January 18, 2018; Accepted: March 22, 2018; Published: March 27, 2018

Citation: Nanda S, Nanda T, Grover D (2018) Complications and Failures of Implants. Oral health case Rep 4: 144. doi:10.4172/2471-8726.1000144

Copyright: $\odot 2018$ Nanda S, et al. This is an open-access article distributed under the terms of the Creative Commons Attribution License, which permits unrestricted use, distribution, and reproduction in any medium, provided the original author and source are credited. 
is an apically repositioned flap and a free gingival graft or connective tissue graft procedure. In the vestibuloplasty, a partial thickness flap is made, repositioned apically, and immobilized by suturing with plain gut to the periosteum. The donor site is prepared usually in palatal mucosa, and either a free gingival graft or connective tissue graft is done as previously described. To cover a large wound area, use two to three narrow bands of palatal mucosa as a graft on the recipient site. This is called a "strip-graft" technique and allows the donor site to heal quickly; there is less discomfort since the large wound area is not exposed completely.

Peri-implant disease: A general category of pathologic changes of the peri-implant tissues. Peri-implant mucositis [5]. inflammatory changes confined to the soft tissue surrounding an implant tissue [5].

Peri-implantitis: radiographically detectable peri-implant bone loss combined with a soft-tissue inflammatory lesion that demonstrates suppration and probing depths $6 \mathrm{~mm}$. The process begins at the coronal aspect of the implant, whereas the more apical portion remains clinically stable (osseointegrated) [6].

Periodontitis and peri-implantitis both involve the loss of crestal bone and the perimucosal seal that is necessary to prevent the apical invasion of bacteria and additional bone loss. Peri-implantitis is an infectious process that involves both hard and soft supporting tissues associated with implants. The etiology of peri-implantitis may be either bacterial or traumatic. The microbiota found around healthy natural teeth and endosseous dental implants are similar, as are the organisms seen in both periodontitis and peri-implantitis. The bacteria and their byproducts must be eliminated from the peri-implant site to arrest further breakdown, particularly if attempts are to be made at regenerating lost bone support.

Efforts at decontaminating the pathologically exposed implant surface fall primarily into one of two broad categories: mechanical and chemotherapeutic.

Mechanical means available for surface cleansing include plastic curettes, rubber prophy cups, and air and powder (sodium bicarbonate) abrasive systems. Metal curettes are to be avoided if no further preparation of the implant surface is planned because they can scratch the implant surface and cause ion transfer.

After mechanical preparation of the implant surface, application of chemotherapeutic agents has been advocated by some groups. Mefferet and Zablotsky et al. $[7,8]$ recommend detoxification of an HA surface

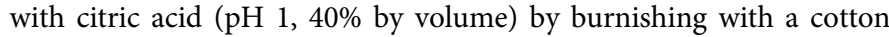
pledget for 30 to 60 second Treatment of a grit-blasted titanium surface with chlor-hexidine, tetracycline, hydrogen peroxide, of; chloramine T was unable to reduce lipopolysaccharide levels significantly below those from burnishing alone with a cotton pledget dipped in sterile saline.

The ailing implant: An implant that may demonstrate bone loss with deeper clinical probing depths but appears to be stable when evaluated at 3- to 4-month intervals. A lamina dura may be present at the borders of the osseous defect, possibly indicating a state of chronicity [7].

The failing implant: An implant that may demonstrate bone loss, increasing clinical probing depths, bleeding on probing, and suppuration. The bone loss may be progressive [7].

The failed implant: An implant that demonstrates clinical mobility, a peri-implant radiolucency, and a dull sound when percussed. A failed implant is nonfunctional and must be removed [7].
Early failures: Failures that occur weeks to a few months after placement Early failures are caused by factors that can interfere with normal healing processes or by an altered host response [9].

Late failures: Failures that arise from pathologic processes that involve a previously osseointegrated implant.

The definitions represent a spectrum of conditions and complications that occur more or less on a time continuum and are not necessarily mutually exclusive. Failures or a failed implant are absolute terms, indicating a hopeless prognosis and the need for removal. Not all failures result from peri-implantitis, and conversely, not all periimplantitis, cases or ailing and failing implants progress to ultimate failure.

\section{Bone Complications}

\section{Progressive marginal bone loss}

Maximum marginal bone loss is approximately 1.0 to $1.5 \mathrm{~mm}$ within the first year after surgery. After about 18 months from the initial surgery, a balance between bone resorption and apposition is reached and fixed at an approximate loss of 0.05 to $0.1 \mathrm{~mm}$ in one year. The total bone loss for a ten-year period of time is less than $1.0 \mathrm{~mm}$ in a normal situation. The cortical-like bone formed around the fixture rarely shows further remodeling or bone loss in radiographic followup [10].

If progressive marginal bone loss is seen radiographically, probable causes including inflammation, occlusal trauma, or prosthetic mechanical complications should be evaluated. If marginal bone loss seems to be caused by local inflammation, appropriate procedures to eliminate the source of inflammation are done with necessary plaque control.

When accelerated bone loss is evident, possible fixture fracture should be investigated. When fixture fracture occurs, it is usually at the neck of the fixture or the apical portion of the fixture at the level of the transverse holes. The incidence rate of fixture fracture is less than $3.5 \%$ [2]. Two radiographs from different angles are needed to evaluate a fixture fracture site.

When a fixture is installed adjacent to a natural tooth, marginal bone loss or vertical bone loss can be due to the periodontal condition of the adjacent teeth. The closer the peridontally involved teeth, the greater chances of having more bone loss at the fixture site.

Throughout treatment, periodontal management is important to preventing further bone loss.

\section{Fixture mobility}

If fixture mobility is detected, regardless of degree of mobility, this is considered a failure to osseointegrate. Fixture mobility can result in fibrous connective tissue formation around the fixture. The fibrous connective tissue becomes thicker with time resulting in increased fixture mobility.

When mobility is detected, the fixture must be extracted. There is no reason or evidence to support further observation because osseointegration is never achieved once it is lost. Under local anesthesia, cut any fibrous tissue attachment, rotate the fixture, then extract it. All nonmineralized connective tissue lining is removed from the fixture site, then the site is closed with a periosteal flap and sutured tightly. With time and new bone formation, it is possible to install another fixture. 
The following are possible causes of fixture mobility and subsequent fixture loss [2]:

- During the first stage surgery, the tissue bed of the fixture site is damaged by aggressive thermal changes during drilling or tapping procedures possibly causing fractures to the bony-threads in the site.

- During the healing period, the surgical site is exposed, and acute inflammation extends to the fixture site.

- Prior to adequate bone healing, occlusal or traumatic forces are transmitted to the fixture.

- Under excessive fixture loading conditions, bony threads fracture in the fixture site.

- Progressive gingivitis inhibits osseointegration.

In contrast, direct bone anchorage to the fixture, early osseointegration, can be achieved under the following conditions:

- In the first stage surgery, minimal surgical trauma is done to the tissue bed, including bone tissue and mucoperiosteal flap.

- Adequate length fixtures are installed carefully into good quality and good quantity types of bone.

- Complete mucoperiosteum closure is achieved without subperiosteal edema.

- Adequate fixture site healing periods elapse.

- No inflammation or infection present at the fixture sites.

- Good occlusal rehabilitation, without the presence of premature contacts or cuspal interferences.

For osseointegration to occur and to help prevent possible fixture mobility, allow for an adequate healing period, have a good fixture site, and good general patient condition. During the healing period radiographs are not needed, especially within the first month after fixture installation. Radiation dosages exceeding 1500 Rads damage immature mesenchymal cells and prevent differentiation in bone cells. If radiographs are absolutely necessary for whatever reason, use a highspeed dental radiographic film and avoid including the fixture site in the radiograph [11].

\section{Discussion}

\section{Mechanical complications}

Component fracture: Fixture fractures require fixture removal with a trephine bur [11]. The trephine bur is a hollow cylinder type bur that cuts surrounding bone for removal of a core which includes the fixture and adjacent bone. If the site is not to be used for another fixture, the remaining fractured fixture can be left within the bone providing the site is free from any pathology.

When the neck of the fixture fractures and osseointegration has been achieved, the fixture is not removed. The fractured portion is duplicated in dental labstone by making an impression of the exposed portion. The U.C.L.A abutment is fitted to the master cast and remaining spaces are waxed to the plastic abutment using inlay wax. Length of the U.C.L.A. abutment is adjusted to the apical end of the gold cylinder and cast in a gold alloy. The abutment is then screwed into the fixture and the prosthesis is connected to the abutment using a self-cure resin.

Abutment screw fracture: Fracture of the abutment screw at the level of the fixture head or neck requires modification for removal of the remaining screw. Cut a groove into the abutment screw fragment and use the smallest drill $(0.5 \mathrm{~mm})$ to rotate the abutment screw fragment out of the fixture. If the abutment screw fragment cannot be removed, splitting the fragment for removal may be difficult and may cause damage to the internal threads of the fixture [11].

Functional speech problems: A fixed partial denture in the anterior maxilla can cause phonetic problems. Normal pronunciation is possible after approximately three months but can take as long as one year for speech recovery. When difficulties occur, removable, artificial gingiva is made of resin or silicone material [12]. Make an impression of the facial surfaces intraorally with an accurate impression material. Make a master cast from the impression using a dental diestone.

Fabrication of the artificial gingiva can be done using a direct sprinkle-on technique and a chemical cure resin. Also, fabrication can be done by completing a wax-up in baseplate wax, following flasking and boil-out procedures, and processing a silicone material. Either method produces artificial gingiva that are placed facially between the prosthesis and residual tissues. Artificial gingiva made from a silicone material can fit well and have good esthetic results, but the material can easily tear and have problems with plaque retention requiring frequent remakes. The artificial gingiva should be cleaned following techniques for removable prostheses.

Esthetic complications in anterior f.p.d.: When the implant prosthesis is employed to replace a small number of teeth in visible areas, management of interdental papilla becomes a concern. Even with a single missing tooth, the flattened papilla adjacent to the edentulous area may require careful surgical reconstruction following the second stage abutment connection. This esthetic plastic surgery may include connective tissue grafting to rebuild the missing papilla tissue. In areas where soft tissue reconstruction is impossible, prosthetic tissue replacement will be required. The difficulty of obtaining aesthetic results increases when multiple teeth are to be replaced with implants. Positional errors occur more frequently for several reasons: 1) there is more freedom for surgeons to place implants; 2) the requirements for each implant site are as stringent for single teeth and the likelihood of multiple ideal implant recipient sites is reduced. The most common errors seen in these types of cases are implants placed in the interproximal areas and differing depth of implant placement. When implants are placed in the interproximal areas it is impossible to obtain an aesthetic result [13].

\section{Conclusion}

Failure of implant is a multifactorial occurrence. A combination of causes leads to ultimate failure of implant. Every dentist needs to identify the cause to treat the present condition. Proper data collection, patient feedback, and accurate diagnostic tools will help point out the reason for failure. An early intervention is always possible if regular checkups are undertaken. The treatment strategy for complications and failing implants is influenced by the identification of the possible etiologic factors. When a diagnosis is established, and possible etiologic factors identified, the causative agent should be eliminated, and treatment attempted as soon as possible.

\section{References}

1. Worthington P, Bolender CL, Taylor TD (1987) The Swedish system of osseointegrated implants: Problems and complications encountered during a four-year trial period. Int J Oral Maxillofac Imp 2: 77-84.

2. Adell R, Lekholm U, Rockler B, Branemark PI (1981) A 15-year studyof osseointegrated implants in the treatment of the edentulous jaw. Int $\mathrm{J}$ Oral Surg 10: $387-416$ 
3. Grupe HE, Warren JR (1956) Repair of gingiva defects by sliding flap operation. J Periodontol 27: 92-95.

4. Carranza Jr FA (1984) Mucogingival surgery. In: Glickman's Clinical periodontolog. Philadelphia: W.B. Saunders, USA. pp. 860-899.

5. Jovanovic AS (1993) The management of peri-implant breakdown around functioning osseointegrated implants. J Periodontol 64: 1176-1183.

6. Mombeilli A, Van Oostem MAC, Schurch E (1987) The microbiota associated with successful or failing osseointegrated titanium implants. Oral Microbio Immunol 2: 145-151.

7. Meffert RM, Langer B, Fritz ME (1992) Dental implants: A review. J Periodontol 63: 859-870.

8. Zablotsky MH, Wittring DE, Diiedrich DL (1992) Fibroblastic growth and attachment on hydroxyapatite coated titanium surfaces following the use of various detoxification modalities. Part II: Contaminated hydroxyapatite. Implant Dent 1: 195-202.

9. Tonetti MS, Schmid J (1994) Pathogenesis of implant failures. Periodontology 2000 4: 127-138.

10. Branemark PI, Hansson BO, Adell R, Breine U, Lindstrom J, et al. (1997) Osseointegrated implants in the treatment of the edenutulous jaw. Experience from a 10-year period. Scand J Plast Reconstr Surg 11 (suppl 16): 60-67.

11. Lekholm U, Adell R, Branemark PI, Branemark PI, Zarb GA, et al. (1985) Tissue-integrated prostheses. Chicago: Quintessence Publishing Co Inc: 211 232

12. Parel SM, Balshi TJ, Sullivan DY, Cardenas ER (1986) Gingival augmentation for osseointegrated implant prosthesis. J Prosthet Dent 56: 208-211.

13. Chee W, Jivraj S (2007) Failures in implant dentistry. Br Dent J 202: 123-129. 